Bull. Korean Math. Soc. 50 (2013), No. 1, pp. 143-159

http://dx.doi.org/10.4134/BKMS.2013.50.1.143

\title{
GLOBAL ATTRACTOR FOR COUPLED TWO-COMPARTMENT GRAY-SCOTT EQUATIONS
}

\author{
Xiaopeng Zhao and Bo LiU
}

\begin{abstract}
This paper is concerned with the long time behavior for the solution semiflow of the coupled two-compartment Gray-Scott equations with the homogeneous Neumann boundary condition on a bounded domain of space dimension $n \leq 3$. Based on the regularity estimates for the semigroups and the classical existence theorem of global attractors, we prove that the equations possesses a global attractor in $H^{k}(\Omega)^{4}(k \geq 0)$ space.
\end{abstract}

\section{Introduction}

In this paper, we study a coupled two-compartment Gray-Scott equation, which is a four-component reaction-diffusion system $[4,9,15]$.

$$
\begin{gathered}
u_{t}=d_{1} \Delta u-(F+k) u+u^{2} v+D_{1}(w-u), \\
v_{t}=d_{2} \Delta v+F(1-v)-u^{2} v+D_{2}(z-v), \\
w_{t}=d_{1} \Delta w-(F+k) w+w^{2} z+D_{1}(u-w), \\
z_{t}=d_{2} \Delta z+F(1-z)-w^{2} z+D_{2}(v-z),
\end{gathered}
$$

for $t>0$, on a bounded domain $\Omega \subset \mathbb{R}^{n}, n \leq 3$, the equations (1.1)-(1.4) have the following boundary conditions

$$
\frac{\partial u}{\partial \nu}(t, x)=\frac{\partial v}{\partial \nu}(t, x)=\frac{\partial w}{\partial \nu}(t, x)=\frac{\partial z}{\partial \nu}(t, x)=0, t>0, x \in \partial \Omega,
$$

and initial conditions

$$
\begin{aligned}
u(0, x) & =u_{0}(x), v(0, x)=v_{0}(x), \\
w(0, x) & =w_{0}(x), z(0, x)=z_{0}(x), x \in \Omega,
\end{aligned}
$$

where $d_{1}, d_{2}, F, k, D_{1}$, and $D_{2}$ are positive constants.

Received July 3, 2011; Revised July 1, 2012.

2010 Mathematics Subject Classification. 35B40, 35B41, 35K20.

Key words and phrases. global attractor, two-compartment Gray-Scott equations, regularity estimates.

This work is supposed by Graduate Innovation Fund of Jilin University (Project 20121059). 
As is well-known, the Gray-Scott model was originally a system of two ordinary differential equations describing the kinetics of cubic autocatalytic chemical or biochemical reactions (see $[5,6,7]$ ). Assume $A$ is an autocatalytic reactant which decays to form a product $P$ in the irreversible reactions shown above, while $B$ is another reactant for which higher concentration beyond a certain level increases the rate of its own removal, then the kinetics describes the following scheme of chemical reactions:

$$
2 A+B \stackrel{k_{1}}{\longrightarrow} 3 A, A \stackrel{k_{1}}{\longrightarrow} P
$$

where $k_{1}$ is the reaction rate constant. Then by the law of mass action and the Fick's law, one obtain a system of two nonlinear reaction-diffusion equations which called the Gray-Scott equations:

$$
\begin{aligned}
& u_{t}=d_{1} \Delta u-(F+k) u+u^{2} v, \\
& v_{t}=d_{2} v+F(1-v)-u^{2} v,
\end{aligned}
$$

where $k$ is called the effective production rate constant and $1 / F$ is called the mean residence time in dimensionless units. The known examples of isothermal autocatalytic reactions which can be modeled by Gray-Scott equations, including the ferrocyanide-iodate-sulphite reaction, the chlorite-iodide-malonic acid (CIMA) reaction, and quite a few enzyme catalytic reactions (see $[1,2,10]$ ). During the past years, there are many papers were denoted to the Gray-Scott equations, for example $[8,13,14]$ and so on.

The dynamic properties of diffusion equation and diffusion system such as the global asymptotical behaviors of solutions and global attractors are important for the study of diffusion model, which ensure the stability of diffusion phenomena and provide the mathematical foundation for the study of diffusion dynamics. There are many studies on the existence of global attractors for diffusion equations. For the classical results we refer the reader to $[3,11,19,21,22,24]$. Recently, based on the iteration technique for regularity estimates, combining with the classical existence theorem of global attractors, Song et al. [17, 18] considered the global attractor for some parabolic equations, such as Cahn-Hilliard equation, Swift-Hohenberg equation and so on, in $H^{k}(0 \leq k \leq \infty)$ space. Zhao and Liu [23] studied the global attractor for a fourth order parabolic equation modeling epitaxial thin-film growth in $H^{k}(0 \leq k<5)$ space.

In this paper, we are interested in the existence of global attractors for the diffusion system (1.1)-(1.4). Based on You's recent paper [20] and Ma and Wang's work [12], we shall prove that the problem (1.1)-(1.6) possesses a global attractor in $H^{k}(\Omega)^{4}(0 \leq k<\infty)$ space.

The outline of this paper is as follows: In the next section, we give some preparations for our consideration, we also give the main result on the existence of global attractor for the problem (1.1)-(1.6); In Section 3, the main result is proved. 
In the following, the letters $C, C_{i},(i=0,1,2, \ldots)$ will always denote positive constants different in various occurrences.

\section{Preliminary}

Assume $X$ and $X_{1}$ are two Banach spaces, $X_{1} \subset X$ a compact and dense inclusion. Consider the following equation defined on $X$,

$$
\left\{\begin{array}{l}
U_{t}=L U+Q U \\
U(0)=U_{0}
\end{array}\right.
$$

where $U$ is an unknown function, $L: X_{1} \rightarrow X$ a linear operator and $Q: X_{1} \rightarrow$ $X$ a nonlinear operator. Then the solution of (2.1) can be expressed as

$$
U\left(t, U_{0}\right)=S(t) U_{0},
$$

where $S(t): X \rightarrow X(t \geq 0)$ is a semiflows generated by (2.1).

We used to assume that the linear operator $L: X_{1} \rightarrow X$ in (2.1) is a sectorial operator, which generates an analytic semiflows $e^{t L}$, and $L$ induces the fractional power operators $\mathscr{L}^{\alpha}$ and fractional order spaces $X_{\alpha}$ as follows,

$$
\mathscr{L}^{\alpha}=(-L)^{\alpha}: X_{\alpha} \rightarrow X, \alpha \in R,
$$

where $X_{\alpha}=D\left(\mathscr{L}^{\alpha}\right)$ is the domain of $\mathscr{L}^{\alpha}$. By the semiflows theory of linear operators, $X_{\beta} \subset X_{\alpha}$ is a compact inclusion for any $\beta>\alpha$. If you want to know more about the space $H_{\alpha}$, I recommend you read [12].

Now, we introduce a lemma on the existence of global attractor which can be founded in $[12,17,18,23]$.

Lemma 2.1. Assume that $U\left(t, U_{0}\right)=S(t) U_{0}\left(U_{0} \in X, t \geq 0\right)$ is a solution of (2.1) and $S(t)$ the semiflows generated by (2.1). Assume further that $X_{\alpha}$ is the fractional order space generated by $L$ and

(B1) For some $\alpha \geq 0$ there is a bounded set $B \subset X_{\alpha}$, which means for any $U_{0} \in X_{\alpha}$, there exists $t_{U_{0}}>0$ such that

$$
U\left(t, U_{0}\right) \in B, \forall t>t_{U_{0}}
$$

(B2) There is a $\beta>\alpha$, for any bounded set $E \subset X_{\beta}, \exists T>0$ and $C>0$ such that

$$
\left\|U\left(t, U_{0}\right)\right\|_{X_{\beta}} \leq C, \forall t>T, U_{0} \in E .
$$

Then (2.1) has a global attractor $\mathscr{A} \subset X_{\alpha}$ which attracts any bounded set of $X_{\alpha}$ in the $X_{\alpha}$-norm.

We also have the following lemma which can be founded in $[12,17,18,23]$.

Lemma 2.2. Assume that $L: X_{1} \rightarrow X_{\alpha}$ is a sectorial operator which generates an analytic semiflows $T(t)=e^{t L}$. If all eigenvalues $\lambda$ of $L$ satisfy $\operatorname{Re} \lambda<-\lambda_{0}$ for some real number $\lambda_{0}>0$, then for $\mathscr{L}^{\alpha}(\mathscr{L}=-L)$ we have

(C1) $T(t): X \rightarrow X_{\alpha}$ is bounded for all $\alpha \in R^{1}$ and $t>0$;

(C2) $T(t) \mathscr{L}^{\alpha} x=\mathscr{L}^{\alpha} T(t) x, \forall x \in X_{\alpha}$; 
(C3) For each $t>0, \mathscr{L}^{\alpha} T(t): X \rightarrow X$ is bounded, and

$$
\left\|\mathscr{L}^{\alpha} T(t)\right\| \leq C_{\alpha} t^{-\alpha} e^{-\delta t}
$$

where some $\delta>0$ and $C_{\alpha}>0$ is a constant depending only on $\alpha$;

(C4) The $X_{\alpha}$-norm can be defined by $\|x\|_{X_{\alpha}}=\left\|\mathscr{L}^{\alpha} x\right\|_{X}$.

Now, we introduce the space as follows

$$
\left\{\begin{array}{l}
\mathcal{H}=H=L^{2}(\Omega) \times L^{2}(\Omega) \times L^{2}(\Omega) \times L^{2}(\Omega) \\
\mathcal{H}_{\frac{1}{2}}=\left\{(u, v, w, z) \in H^{1}(\Omega) \times H^{1}(\Omega) \times H^{1}(\Omega) \times H^{1}(\Omega)\right. \\
\left.\quad \frac{\partial u}{\partial \nu}=\frac{\partial v}{\partial \nu}=\frac{\partial w}{\partial \nu}=\frac{\partial z}{\partial \nu}=0\right\} \\
\mathcal{H}_{1}=\left(H^{2}(\Omega) \times H^{2}(\Omega) \times H^{2}(\Omega) \times H^{2}(\Omega)\right) \cap \mathcal{H}_{\frac{1}{2}}
\end{array}\right.
$$

We also define the operators $L_{i}(i=1,2)$ and $Q_{i}(i=1,2,3,4)$ by

$$
\left\{\begin{array}{l}
L_{1} u=d_{1} \Delta u, L_{2} v=d_{2} \Delta v, L_{1} w=d_{1} \Delta w, L_{2} z=d_{2} \Delta z \\
Q_{1} g=q_{1}(u, v, w, z)=-(F+k) u+u^{2} v+D_{1}(w-u) \\
Q_{2} g=q_{2}(u, v, w, z)=F(1-v)-u^{2} v+D_{2}(z-v) \\
Q_{3} g=q_{3}(u, v, w, z)=-(F+k) w+w^{2} z+D_{1}(u-w) \\
Q_{4} g=q_{4}(u, v, w, z)=F(1-z)-w^{2} z+D_{2}(v-z)
\end{array}\right.
$$

where $g=\operatorname{col}(u, v, w, z)$. It is easy to check that $q_{i}(i=1,2,3,4)$ are nonlinear functions, $q_{1}(u, v, w, z)=q_{3}(w, z, u, v)$ and $q_{2}(u, v, w, z)=q_{4}(w, z, u, v)$. Obviously, the linear operator $L_{i}: H^{2}(\Omega) \rightarrow L^{2}(\Omega),(i=1,2)$ given by $(2.4)$ are sectorial operators.

By using the Lumer-Phillips theorem and the analytic semiflows generation theorem [16], we obtain the linear operator

$$
L=\left(\begin{array}{rrrr}
d_{1} \Delta & 0 & 0 & 0 \\
0 & d_{2} \Delta & 0 & 0 \\
0 & 0 & d_{1} \Delta & 0 \\
0 & 0 & 0 & d_{2} \Delta
\end{array}\right)=\left(\begin{array}{c}
L_{1} \\
L_{2} \\
L_{1} \\
L_{2}
\end{array}\right): H^{2}(\Omega)^{4} \rightarrow L^{2}(\Omega)^{4}
$$

which is the generator of an analytic $C_{0}$-semiflows on the Hilbert space $L^{2}(\Omega)^{4}$. Define

$$
Q g=\left(\begin{array}{l}
Q_{1} g \\
Q_{2} g \\
Q_{3} g \\
Q_{4} g
\end{array}\right)=\left(\begin{array}{l}
q_{1}(u, v, w, z) \\
q_{2}(u, v, w, z) \\
q_{3}(u, v, w, z) \\
q_{4}(u, v, w, z)
\end{array}\right)=\left(\begin{array}{l}
-(F+k) u+u^{2} v+D_{1}(w-u) \\
F(1-v)-u^{2} v+D_{2}(z-v) \\
-(F+k) w+w^{2} z+D_{1}(u-w) \\
F(1-z)-w^{2} z+D_{2}(v-z)
\end{array}\right)
$$

then the initial boundary value problem (1.1)-(1.6) is formulated into the following problem:

$$
\frac{d g}{d t}=L g+Q g, t>0
$$

where $g=\operatorname{col}(u, v, w, z)$, or written as $(u, v, w, z)$, for any initial data $g(0)=$ $g_{0}=\operatorname{col}\left(u_{0}, v_{0}, w_{0}, z_{0}\right)$, or written as $\left(u_{0}, v_{0}, w_{0}, z_{0}\right)$. It is easy to see that in (2.6) $L$ is a linear operator and $Q$ a nonlinear operator. 
Compared with (2.1), it is easy to see that $X=\mathcal{H}, X_{1}=\mathcal{H}_{1}, L: \mathcal{H}_{1} \rightarrow \mathcal{H}$ is a linear sectorial operator and $Q$ a nonlinear operator in (2.6). We can define the fractional order spaces $\mathscr{L}^{\alpha}$ as $(2.2)$, where $\mathcal{H}_{\alpha}=D\left(\mathscr{L}^{\alpha}\right)=\mathrm{H}_{\alpha} \times \mathrm{H}_{\alpha} \times$ $\mathrm{H}_{\alpha} \times \mathrm{H}_{\alpha}=D\left(\left(-L_{1}\right)^{\alpha}\right) \times D\left(\left(-L_{2}\right)^{\alpha}\right) \times D\left(\left(-L_{1}\right)^{\alpha}\right) \times D\left(\left(-L_{2}\right)^{\alpha}\right)$ is the domain of $\mathscr{L}^{\alpha}$.

The following propositions on the existence and uniqueness of strong solution and global weak solution for problem (2.6) can be found in [20].

Proposition 2.3. For any given initial data $g_{0} \in \mathcal{H}$, there exists a unique, local weak solution $g(t)=(u(t), v(t), w(t), z(t)), t \in[0, \tau]$ for some $\tau>0$, of the problem (2.6), which becomes a strong solution on $(0, \tau]$ and satisfies

$$
g \in C\left(\left[0, T_{\max }\right) ; \mathcal{H}\right) \bigcap C^{1}\left(\left(0, T_{\max }\right) ; \mathcal{H}\right) \bigcap L^{2}\left(0, T_{\max } ; \mathcal{H}_{\frac{1}{2}}\right) .
$$

Proposition 2.4. For any given initial data $g_{0} \in \mathcal{H}$, there exists a unique, global, weak solution $g(t)=(u(t), v(t), w(t), z(t)), t \in[0, \infty)$, of the problem (2.6).

Based on Proposition 2.4, we can define a semiflow $\{S(t)\}_{t \geq 0}$ on $L^{2}(\Omega)^{4}$, where

$$
S(t): g_{0} \longmapsto g\left(t, g_{0}\right), g_{0} \in L^{2}(\Omega)^{4}, t \geq 0,
$$

which will be called coupled Gray-Scott semiflow generated by the two-compartment Gray-Scott evolutionary equations (1.1)-(1.6).

We summarize the results in [20].

Proposition 2.5. For any given positive parameters $d_{1}, d_{2}, F, k, D_{1}$ and $D_{2}$, there exists a constant $K_{1}>0$ such that the set

$$
B_{0}=\left\{g \in L^{2}(\Omega)^{4}:\|g\|^{2} \leq K_{1}\right\}
$$

is a bounded absorbing set in $L^{2}(\Omega)^{4}$ for the coupled Gray-Scott semiflow $\{S(t)\}_{t \geq 0}$.

Proposition 2.6. For any given positive parameters $d_{1}, d_{2}, F, k, D_{1}, D_{2}$ and initial data $\left(u_{0}, v_{0}, w_{0}, z_{0}\right) \in B_{0}$, the $(u(t), w(t))$ components of the solution trajectory $g(t)=S(t) g_{0}$ of the initial value problem (2.6) satisfy

$$
\|u(t)\|_{H^{1}}^{2}+\|w(t)\|_{H^{1}}^{2} \leq M_{1}, \forall t>T_{1},
$$

where $M_{1}>0$ is a constant depending on $|\Omega|$ but independent of the initial data, and $T_{1}>0$ is finite and only depends on $K_{1}$ and $|\Omega|$.

Proposition 2.7. For any given positive parameters $d_{1}, d_{2}, F, k, D_{1}, D_{2}$ and initial data $\left(u_{0}, v_{0}, w_{0}, z_{0}\right) \in B_{0}$, the $(v(t), z(t))$ components of the solution trajectory $g(t)=S(t) g_{0}$ of the initial value problem (2.6) satisfy

$$
\|v(t)\|_{H^{1}}^{2}+\|z(t)\|_{H^{1}}^{2} \leq M_{2}, \forall t>T_{2},
$$

where $M_{2}>0$ is a constant depending on $|\Omega|$ but independent of the initial data, and $T_{2}>0$ is finite and only depends on $K_{1}$ and $|\Omega|$. 
Proposition 2.8. For any given positive parameters $d_{1}, d_{2}, F, k, D_{1}, D_{2}$ and the constant $R>0$, there exists a constant $M(R)>0$ such that if the initial datum $\left(u_{0}, v_{0}, w_{0}, z_{0}\right) \in H^{1}(\Omega)^{4}$ and

$$
\left\|u_{0}\right\|_{H^{1}(\Omega)}^{2}+\left\|v_{0}\right\|_{H^{1}(\Omega)}^{2}+\left\|w_{0}\right\|_{H^{1}(\Omega)}^{2}+\left\|z_{0}\right\|_{H^{1}(\Omega)}^{2} \leq R
$$

then for all $t \geq 0$, we have

$$
\|u\|_{H^{1}(\Omega)}^{2}+\|v\|_{H^{1}(\Omega)}^{2}+\|w\|_{H^{1}(\Omega)}^{2}+\|z\|_{H^{1}(\Omega)}^{2} \leq M(R) .
$$

Proposition 2.9. For any given positive parameters $d_{1}, d_{2}, F, k, D_{1}$ and $D_{2}$, there exists a global attractor in the phase space $L^{2}(\Omega)^{4}$ for the solution semiflow $\{S(t)\}_{t \geq 0}$ of the problem (2.5).

In the following, we give the main result, which provides the existence of global attractors of the equations (1.1)-(1.4) in any $k$ th space $H^{k}(\Omega)^{4}$, where $0 \leq k<\infty$.

Theorem 2.10. For any given positive parameters $d_{1}, d_{2}, F, k, D_{1}, D_{2}$ and $g_{0}=$ $\left(u_{0}, v_{0}, w_{0}, z_{0}\right) \in \mathcal{H}_{\alpha}(\forall \alpha \geq 0)$, the semiflows $S(t)$ associated with problem (1.1)-(1.6) possesses a global attractor $\mathscr{A}$ in $\mathcal{H}_{\alpha}$ space and $\mathscr{A}$ attractors any bounded set of $\mathcal{H}_{\alpha}$ in the $\mathcal{H}_{\alpha}$-norm.

\section{Proof of Theorem 2.10}

We are now in a position to state and prove the main theorem in this paper, which provides the existence of a global attractor of the equations (1.1)-(1.6) in spaces $\mathcal{H}_{\alpha}$ of any $\alpha$ th differentiable function.

For any $g_{0}=\left(u_{0}, v_{0}, w_{0}, z_{0}\right) \in \mathcal{H}$, the solution $(u, v, w, z)$ of the problem (1.1)-(1.6) can be written as

$$
\begin{aligned}
u\left(t, u_{0}\right) & =e^{t L_{1}} u_{0}+\int_{0}^{t} e^{(t-\tau) L_{1}} Q_{1} g d \tau \\
& =e^{t L_{1}} u_{0}+\int_{0}^{t} e^{(t-\tau) L_{1}} q_{1}(u, v, w, z) d \tau \\
v\left(t, v_{0}\right) & =e^{t L_{2}} v_{0}+\int_{0}^{t} e^{(t-\tau) L_{2}} Q_{2} g d \tau \\
& =e^{t L_{2}} v_{0}+\int_{0}^{t} e^{(t-\tau) L_{2}} q_{2}(u, v, w, z) d \tau, \\
w\left(t, w_{0}\right) & =e^{t L_{1}} w_{0}+\int_{0}^{t} e^{(t-\tau) L_{1}} Q_{3} g d \tau \\
& =e^{t L_{1}} w_{0}+\int_{0}^{t} e^{(t-\tau) L_{1}} q_{3}(u, v, w, z) d \tau, \\
z\left(t, z_{0}\right) & =e^{t L_{2}} z_{0}+\int_{0}^{t} e^{(t-\tau) L_{2}} Q_{4} g d \tau
\end{aligned}
$$




$$
=e^{t L_{2}} z_{0}+\int_{0}^{t} e^{(t-\tau) L_{2}} q_{4}(u, v, w, z) d \tau,
$$

By Lemma 2.1, in order to prove Theorem 2.10, we first prove the following lemma.

Lemma 3.1. Suppose $d_{1}, d_{2}, F, k, D_{1}$ and $D_{2}$ are given positive parameters, $g=(u, v, w, z)$ is a solution to the problem (1.1)-(1.6), $g_{0}=\left(u_{0}, v_{0}, w_{0}, z_{0}\right) \in$ $\mathcal{H}_{\alpha}(\forall \alpha \geq 0)$, then, the semiflows $S(t)$ associated with problem (1.1)-(1.6) is uniformly compact in $\mathcal{H}_{\alpha}$.

Proof. It suffices to prove that for any bounded set $B \subset \mathcal{H}_{\alpha}$ with initial value $g_{0}=\left(u_{0}, v_{0}, w_{0}, z_{0}\right) \in B \subset \mathcal{H}_{\alpha}$, there exists $C>0$ such that

$$
\left\|g\left(u, g_{0}\right)\right\|_{\mathcal{H}_{\alpha}} \leq C, \forall t \geq 0, \alpha \geq 0 .
$$

Obviously, if we get

$\left\|u\left(t, u_{0}\right)\right\|_{\mathrm{H}_{\alpha}}^{2}+\left\|v\left(t, v_{0}\right)\right\|_{\mathrm{H}_{\alpha}}^{2}+\left\|w\left(t, w_{0}\right)\right\|_{\mathrm{H}_{\alpha}}^{2}+\left\|z\left(t, z_{0}\right)\right\|_{\mathrm{H}_{\alpha}}^{2} \leq C . \forall t \geq 0, \alpha \geq 0$, then, we obtain (3.5) immediately.

For $\alpha=\frac{1}{2}$, this follows from Proposition 2.8, i.e., for any bounded set $B \subset \mathcal{H}_{\frac{1}{2}}$ with initial value $\left(u_{0}, v_{0}, w_{0}, z_{0}\right) \in B \subset \mathcal{H}_{\frac{1}{2}}$, there exists a constant $C>0$ such that $\forall t \geq 0$,

$$
\left\|u\left(t, u_{0}\right)\right\|_{\mathrm{H}_{\frac{1}{2}}}+\left\|v\left(t, v_{0}\right)\right\|_{\mathrm{H}_{\frac{1}{2}}}+\left\|w\left(t, w_{0}\right)\right\|_{\mathrm{H}_{\frac{1}{2}}}+\left\|z\left(t, z_{0}\right)\right\|_{\mathrm{H}_{\frac{1}{2}}} \leq C .
$$

So, we only need to show (3.5) for any $\alpha \geq \frac{1}{2}$. There are three steps for us to prove it.

Step 1 . We prove that for any bounded set $B \subset \mathcal{H}_{\alpha}\left(\frac{1}{2} \leq \alpha<1\right)$, there exists a positive constant $C$ such that $\forall t \geq 0, \frac{1}{2} \leq \alpha<1$,

$$
\left\|u\left(t, u_{0}\right)\right\|_{\mathrm{H}_{\alpha}}^{2}+\left\|v\left(t, v_{0}\right)\right\|_{\mathrm{H}_{\alpha}}^{2}+\left\|w\left(t, w_{0}\right)\right\|_{\mathrm{H}_{\alpha}}^{2}+\left\|z\left(t, z_{0}\right)\right\|_{\mathrm{H}_{\alpha}}^{2} \leq C .
$$

For the dimension $n \leq 3$, we have $H^{1}(\Omega) \hookrightarrow L^{6}(\Omega)$. It then follows from Proposition 2.5 and Proposition 2.8 that

$$
\begin{aligned}
\left\|q_{1}(u, v, w, z)\right\|_{L^{2}}^{2} & =\int_{\Omega}\left|q_{1}(u, v, w, z)\right|^{2} d x \\
& =\int_{\Omega}\left[-(F+k) u+u^{2} v+D_{1}(w-u)\right]^{2} d x \\
& \leq C\left(\int_{\Omega} u^{2} d x+\int_{\Omega} w^{2} d x+\int_{\Omega} u^{4} v^{2} d x\right) \\
& \leq C\left(\int_{\Omega} u^{2} d x+\int_{\Omega} w^{2} d x+\left(\int_{\Omega} u^{6} d x\right)^{\frac{4}{3}}+\left(\int_{\Omega} v^{6} d x\right)^{\frac{2}{3}}\right) \\
& \leq C\left(\|u\|^{2}+\|w\|^{2}+\|u\|_{H^{1}}^{\frac{8}{3}}+\|v\|_{H^{1}}^{\frac{4}{3}}\right) \leq C
\end{aligned}
$$

and

$\left\|q_{2}(u, v, w, z)\right\|_{L^{2}}^{2}=\int_{\Omega}\left|q_{2}(u, v, w, z)\right|^{2} d x$ 


$$
\begin{aligned}
& =\int_{\Omega}\left[F(1-v)-u^{2} v+D_{2}(z-v)\right]^{2} d x \\
& \leq C\left(C+\int_{\Omega} v^{2} d x+\int_{\Omega} u^{4} v^{2} d x+\int_{\Omega} z^{2} d x\right) \\
& \leq C\left(C+\int_{\Omega} v^{2} d x+\left(\int_{\Omega} u^{6} d x\right)^{\frac{4}{3}}+\left(\int_{\Omega} v^{6} d x\right)^{\frac{2}{3}}+\int_{\Omega} z^{2} d x\right) \\
& \leq C\left(\|v\|^{2}+\|z\|^{2}+\|u\|_{H^{1}}^{\frac{8}{3}}+\|v\|_{H^{1}}^{\frac{4}{3}}\right) \leq C .
\end{aligned}
$$

Note that $q_{1}(u, v, w, z)=q_{3}(w, z, u, v)$ and $q_{2}(u, v, w, z)=q_{4}(w, z, u, v)$, simple calculations show that

$$
\begin{gathered}
\left\|q_{3}(u, v, w, z)\right\|_{L^{2}}^{2} \leq C, \\
\left\|q_{4}(u, v, w, z)\right\|_{L^{2}}^{2} \leq C .
\end{gathered}
$$

By (3.1), (3.6) and (3.8), we obtain

$$
\begin{aligned}
\left\|u\left(t, u_{0}\right)\right\|_{\mathrm{H}_{\alpha}} & =\left\|e^{t L_{1}} u_{0}+\int_{0}^{t} e^{(t-\tau) L_{1}} q_{1}(u, v, w, z) d \tau\right\|_{\mathrm{H}_{\alpha}} \\
& \leq\left\|e^{t L_{1}} u_{0}\right\|_{\mathrm{H}_{\alpha}}+\left\|\int_{0}^{t} e^{(t-\tau) L_{1}} q_{1}(u, v, w, z) d \tau\right\|_{\mathrm{H}_{\alpha}} \\
& \leq C\left\|u_{0}\right\|_{\mathrm{H}_{\alpha}}+\int_{0}^{t}\left\|\left(-L_{1}\right)^{\alpha} e^{(t-\tau) L_{1}}\right\| \cdot\left\|q_{1}(u, v, w, z)\right\|_{L^{2}(\Omega)} d \tau \\
& \leq C\left\|u_{0}\right\|_{\mathrm{H}_{\alpha}}+C \int_{0}^{t} \tau^{-\alpha} e^{-\delta \tau} d \tau \\
& \leq C, \forall t \geq 0, g_{0} \in B,
\end{aligned}
$$

where $0<\alpha<1$. By (3.2), (3.6) and (3.9), we obtain

$$
\begin{aligned}
\left\|v\left(t, v_{0}\right)\right\|_{\mathrm{H}_{\alpha}} & =\left\|e^{t L_{2}} v_{0}+\int_{0}^{t} e^{(t-\tau) L_{2}} q_{2}(u, v, w, z) d \tau\right\|_{\mathrm{H}_{\alpha}} \\
& \leq\left\|e^{t L_{2}} v_{0}\right\|_{\mathrm{H}_{\alpha}}+\left\|\int_{0}^{t} e^{(t-\tau) L_{2}} q_{2}(u, v, w, z) d \tau\right\|_{\mathrm{H}_{\alpha}} \\
& \leq C\left\|v_{0}\right\|_{\mathrm{H}_{\alpha}}+\int_{0}^{t}\left\|\left(-L_{2}\right)^{\alpha} e^{(t-\tau) L_{2}}\right\| \cdot\left\|q_{2}(u, v, w, z)\right\|_{L^{2}(\Omega)} d \tau \\
& \leq C\left\|v_{0}\right\|_{\mathrm{H}_{\alpha}}+C \int_{0}^{t} \tau^{-\alpha} e^{-\delta \tau} d \tau \\
& \leq C, \forall t \geq 0, g_{0} \in B,
\end{aligned}
$$

where $0<\alpha<1$. By (3.3), (3.4), (3.6), (3.10) and (3.11), simple calculations shows that

$$
\begin{gathered}
\left\|w\left(t, w_{0}\right)\right\|_{\mathrm{H}_{\alpha}} \leq C, \forall t \geq 0, g_{0} \in B, \\
\left\|z\left(t, z_{0}\right)\right\|_{\mathrm{H}_{\alpha}} \leq C, \forall t \geq 0, g_{0} \in B,
\end{gathered}
$$


where $0<\alpha<1$. By (3.12), (3.13), (3.14) and (3.15), we obtain (3.7) immediately.

Step 2. We prove that for any bounded set $B \subset \mathcal{H}_{\alpha}\left(1 \leq \alpha<\frac{3}{2}\right)$, there exists a positive constant $C$ such that $\forall t \geq 0,1 \leq \alpha<\frac{3}{2}$,

$$
\left\|u\left(t, u_{0}\right)\right\|_{\mathrm{H}_{\alpha}}^{2}+\left\|v\left(t, v_{0}\right)\right\|_{\mathrm{H}_{\alpha}}^{2}+\left\|w\left(t, w_{0}\right)\right\|_{\mathrm{H}_{\alpha}}^{2}+\left\|z\left(t, z_{0}\right)\right\|_{\mathrm{H}_{\alpha}}^{2} \leq C .
$$

By Proposition 2.5, Proposition 2.8 and the following embedding theorems of fractional order spaces

$$
\mathrm{H}_{\alpha} \hookrightarrow C^{0}(\Omega) \bigcap H^{1}(\Omega), \forall \alpha>\frac{3}{4},
$$

we obtain

(3.18) $\left\|q_{1}(u, v, w, z)\right\|_{\mathrm{H}_{\frac{1}{2}}}^{2}$

$$
\begin{aligned}
\leq & C \int_{\Omega}\left|\nabla q_{1}(u, v, w, z)\right|^{2} d x+C_{0} \\
\leq & C \int_{\Omega}\left|\nabla\left(-(F+k) u+u^{2} v+D_{1}(w-u)\right)\right|^{2} d x+C_{0} \\
\leq & C \int_{\Omega}\left(-(F+k) \nabla u+2 u v \nabla u+u^{2} \nabla v+D_{1} \nabla w-D_{1} \nabla u\right)^{2} d x+C_{0} \\
\leq & C \int_{\Omega}\left(|\nabla u|^{2}+u^{2} v^{2}|\nabla u|^{2}+u^{4}|\nabla v|^{2}+|\nabla w|^{2}+|\nabla u|^{2}\right) d x+C_{0} \\
\leq & C \int_{\Omega}\left(|\nabla u|^{2}+\sup _{x \in \Omega} u^{2} v^{2} \cdot|\nabla u|^{2}+\sup _{x \in \Omega} u^{4} \cdot|\nabla v|^{2}+|\nabla w|^{2}+|\nabla u|^{2}\right) d x \\
& +C_{0} \\
\leq & C \int_{\Omega}\left(|\nabla u|^{2}+|\nabla u|^{2}+|\nabla v|^{2}+|\nabla w|^{2}+|\nabla u|^{2}\right) d x+C_{0} \\
\leq & C\left(\|u\|_{\mathrm{H}_{\alpha}}^{2}+\|v\|_{\mathrm{H}_{\alpha}}^{2}+\|w\|_{\mathrm{H}_{\alpha}}^{2}\right)+C_{0} \leq C
\end{aligned}
$$

and

$$
\begin{aligned}
& \left\|q_{2}(u, v, w, z)\right\|_{\mathrm{H}_{\frac{1}{2}}}^{2} \\
\leq & C \int_{\Omega}\left|\nabla q_{2}(u, v, w, z)\right|^{2} d x+C_{0} \\
\leq & C \int_{\Omega}\left[\nabla\left(F(1-v)-u^{2} v+D_{2}(z-v)\right)\right]^{2} d x+C_{0} \\
\leq & C \int_{\Omega}\left(-F \nabla v-2 u v \nabla u-u^{2} \nabla v+D_{2} \nabla z-D_{2} \nabla v\right)^{2} d x+C_{0} \\
\leq & C \int_{\Omega}\left(|\nabla v|^{2}+u^{2} v^{2}|\nabla u|^{2}+u^{4}|\nabla v|^{2}+|\nabla z|^{2}+|\nabla v|^{2}\right) d x+C_{0} \\
\leq & C \int_{\Omega}\left(|\nabla v|^{2}+\sup _{x \in \Omega} u^{2} v^{2} \cdot|\nabla u|^{2}+\sup _{x \in \Omega} u^{4} \cdot|\nabla v|^{2}+|\nabla z|^{2}+|\nabla v|^{2}\right) d x
\end{aligned}
$$




$$
\begin{aligned}
& +C_{0} \\
\leq & C \int_{\Omega}\left(|\nabla v|^{2}+|\nabla u|^{2}+|\nabla v|^{2}+|\nabla z|^{2}+|\nabla v|^{2}\right) d x+C_{0} \\
\leq & C\left(\|u\|_{\mathrm{H}_{\alpha}}^{2}+\|v\|_{\mathrm{H}_{\alpha}}^{2}+\|z\|_{\mathrm{H}_{\alpha}}^{2}\right)+C_{0} \leq C .
\end{aligned}
$$

Note that $q_{1}(u, v, w, z)=q_{3}(w, z, u, v)$ and $q_{2}(u, v, w, z)=q_{4}(w, z, u, v)$, simple calculations show that

$$
\begin{gathered}
\left\|q_{3}(u, v, w, z)\right\|_{\mathrm{H}_{\frac{1}{2}}}^{2} \leq C, \\
\left\|q_{4}(u, v, w, z)\right\|_{\mathrm{H}_{\frac{1}{2}}}^{2} \leq C .
\end{gathered}
$$

By (3.1), (3.7) and (3.18), we obtain

$$
\begin{aligned}
\left\|u\left(t, u_{0}\right)\right\|_{\mathrm{H}_{\alpha}} & =\left\|e^{t L_{1}} u_{0}+\int_{0}^{t} e^{(t-\tau) L_{1}} q_{1}(u, v, w, z) d \tau\right\|_{\mathrm{H}_{\alpha}} \\
& \leq\left\|e^{t L_{1}} u_{0}\right\|_{\mathrm{H}_{\alpha}}+\left\|\int_{0}^{t} e^{(t-\tau) L_{1}} q_{1}(u, v, w, z) d \tau\right\|_{\mathrm{H}_{\alpha}} \\
& \leq C\left\|u_{0}\right\|_{\mathrm{H}_{\alpha}}+\int_{0}^{t}\left\|\left(-L_{1}\right)^{-\frac{1}{2}+\alpha} e^{(t-\tau) L_{1}}\right\| \cdot\left\|q_{1}(u, v, w, z)\right\|_{\mathrm{H}_{\frac{1}{2}}} d \tau \\
& \leq C\left\|u_{0}\right\|_{\mathrm{H}_{\alpha}}+C \int_{0}^{t} \tau^{\frac{1}{2}-\alpha} e^{-\delta \tau} d \tau \\
& \leq C, \forall t \geq 0, g_{0} \in B,
\end{aligned}
$$

where $\frac{1}{2}<\alpha<\frac{3}{2}$. By (3.2), (3.7) and (3.19), we obtain

$$
\begin{aligned}
\left\|v\left(t, v_{0}\right)\right\|_{\mathrm{H}_{\alpha}} & =\left\|e^{t L_{2}} v_{0}+\int_{0}^{t} e^{(t-\tau) L_{2}} q_{2}(u, v, w, z) d \tau\right\|_{\mathrm{H}_{\alpha}} \\
& \leq\left\|e^{t L_{2}} v_{0}\right\|_{\mathrm{H}_{\alpha}}+\left\|\int_{0}^{t} e^{(t-\tau) L_{2}} q_{2}(u, v, w, z) d \tau\right\|_{\mathrm{H}_{\alpha}} \\
& \leq C\left\|v_{0}\right\|_{\mathrm{H}_{\alpha}}+\int_{0}^{t}\left\|\left(-L_{2}\right)^{-\frac{1}{2}+\alpha} e^{(t-\tau) L_{2}}\right\| \cdot\left\|q_{2}(u, v, w, z)\right\|_{\mathrm{H}_{\frac{1}{2}}} d \tau \\
& \leq C\left\|v_{0}\right\|_{\mathrm{H}_{\alpha}}+C \int_{0}^{t} \tau^{\frac{1}{2}-\alpha} e^{-\delta \tau} d \tau \\
& \leq C, \forall t \geq 0, g_{0} \in B
\end{aligned}
$$

where $\frac{1}{2}<\alpha<\frac{3}{2}$. By (3.3), (3.4), (3.7), (3.20) and (3.21), simple calculations shows that

$$
\begin{gathered}
\left\|w\left(t, w_{0}\right)\right\|_{\mathrm{H}_{\alpha}} \leq C, \forall t \geq 0, g_{0} \in B, \\
\left\|z\left(t, z_{0}\right)\right\|_{\mathrm{H}_{\alpha}} \leq C, \forall t \geq 0, g_{0} \in B,
\end{gathered}
$$

where $\frac{1}{2}<\alpha<\frac{3}{2}$. By (3.22), (3.23), (3.24) and (3.25), we obtain (3.16) immediately. 
Step 3. We prove that for any bounded set $B \subset \mathcal{H}_{\alpha}\left(\frac{3}{2} \leq \alpha<2\right)$, there exists a positive constant $C$ such that $\forall t \geq 0, \frac{3}{2} \leq \alpha<2$,

$$
\left\|u\left(t, u_{0}\right)\right\|_{\mathrm{H}_{\alpha}}^{2}+\left\|v\left(t, v_{0}\right)\right\|_{\mathrm{H}_{\alpha}}^{2}+\left\|w\left(t, w_{0}\right)\right\|_{\mathrm{H}_{\alpha}}^{2}+\left\|z\left(t, z_{0}\right)\right\|_{\mathrm{H}_{\alpha}}^{2} \leq C .
$$

By Proposition 2.5 and Proposition 2.8 and the following embedding theorems of fractional order spaces

$$
\mathrm{H}_{\alpha} \hookrightarrow H^{2}(\Omega), H^{2}(\Omega) \hookrightarrow C^{0}(\Omega) \bigcap W^{1,4}(\Omega), \forall \alpha>1,
$$

we obtain

$$
\begin{aligned}
& \left\|q_{1}(u, v, w, z)\right\|_{\mathrm{H}_{1}}^{2} \\
\leq & C \int_{\Omega}\left|\Delta q_{1}(u, v, w, z)\right|^{2} d x+C_{0} \\
\leq & C \int_{\Omega}\left|\Delta\left(-(F+k) u+u^{2} v+D_{1}(w-u)\right)\right|^{2} d x+C_{0} \\
\leq & C \int_{\Omega}\left(-(F+k) \Delta u+2 u v \Delta u+2 v|\nabla u|^{2}+4 u \nabla u \nabla v\right. \\
& \left.+u^{2} \Delta v+D_{1} \Delta w-D_{1} \Delta u\right)^{2} d x+C_{0} \\
\leq & C \int_{\Omega}\left(|\Delta u|^{2}+u^{2} v^{2}|\Delta u|^{2}+v^{2}|\nabla u|^{4}+u^{2}|\nabla u \nabla v|^{2}\right. \\
& \left.+u^{4}|\Delta v|^{2}+|\Delta w|^{2}+|\Delta u|^{2}\right) d x+C_{0} \\
\leq & C \int_{\Omega}\left(|\Delta u|^{2}+\sup _{x \in \Omega} u^{2} v^{2} \cdot|\Delta u|^{2}+\sup _{x \in \Omega} v^{2} \cdot|\nabla u|^{4}\right. \\
& \left.+\sup _{x \in \Omega} u^{2} \cdot|\nabla u \nabla v|^{2}+\sup _{x \in \Omega} u^{4} \cdot|\Delta v|^{2}+|\Delta w|^{2}+|\Delta u|^{2}\right) d x+C_{0} \\
\leq & C \int_{\Omega}\left(|\Delta u|^{2}+|\nabla v|^{4}+|\nabla u|^{4}+|\Delta v|^{2}+|\Delta w|^{2}\right) d x+C_{0} \\
\leq & C\left(\|u\|_{\mathrm{H}_{\alpha}}^{2}+\|v\|_{\mathrm{H}_{\alpha}}^{4}+\|u\|_{\mathrm{H}_{\alpha}}^{4}+\|v\|_{\mathrm{H}_{\alpha}}^{2}+\|w\|_{\mathrm{H}_{\alpha}}^{2}\right)+C_{0} \leq C
\end{aligned}
$$

and

$$
\begin{aligned}
& \left\|q_{2}(u, v, w, z)\right\|_{\mathrm{H}_{1}}^{2} \\
\leq & C \int_{\Omega}\left|\Delta q_{2}(u, v, w, z)\right|^{2} d x+C_{0} \\
\leq & C \int_{\Omega}\left[\Delta\left(F(1-v)-u^{2} v+D_{2}(z-v)\right)\right]^{2} d x+C_{0} \\
\leq & C \int_{\Omega}\left(-F \Delta v-2 u v \Delta u-2 v|\nabla u|^{2}-4 u \nabla u \nabla v\right. \\
& \left.-u^{2} \Delta v+D_{2} \Delta z-D_{2} \Delta v\right)^{2} d x+C_{0} \\
\leq & C \int_{\Omega}\left(|\Delta u|^{2}+u^{2} v^{2}|\Delta u|^{2}+v^{2}|\nabla u|^{4}+u^{2}|\nabla u \nabla v|^{2}\right.
\end{aligned}
$$




$$
\begin{aligned}
& \left.+u^{4}|\Delta v|^{2}+|\Delta z|^{2}+|\Delta v|^{2}\right) d x+C_{0} \\
\leq & C \int_{\Omega}\left(|\Delta u|^{2}+\sup _{x \in \Omega} u^{2} v^{2} \cdot|\Delta u|^{2}+\sup _{x \in \Omega} v^{2} \cdot|\nabla u|^{4}\right. \\
& \left.+\sup _{x \in \Omega} u^{2} \cdot|\nabla u \nabla v|^{2}+\sup _{x \in \Omega} u^{4} \cdot|\Delta v|^{2}+|\Delta z|^{2}+|\Delta v|^{2}\right) d x+C_{0} \\
\leq & C \int_{\Omega}\left(|\Delta u|^{2}+|\nabla u|^{4}+|\nabla v|^{4}+|\Delta v|^{2}+|\Delta z|^{2}\right) d x+C_{0} \\
\leq & C\left(\|\Delta u\|^{2}+\|\Delta v\|^{2}+\|\nabla u\|^{4}+\|\nabla v\|^{4}+\|\Delta z\|^{2}\right)+C_{0}
\end{aligned}
$$

Note that $q_{1}(u, v, w, z)=q_{3}(w, z, u, v)$ and $q_{2}(u, v, w, z)=q_{4}(w, z, u, v)$, simple calculations show that

$$
\begin{aligned}
& \left\|q_{3}(u, v, w, z)\right\|_{\mathrm{H}_{1}}^{2} \leq C, \\
& \left\|q_{4}(u, v, w, z)\right\|_{\mathrm{H}_{1}}^{2} \leq C .
\end{aligned}
$$

By (3.1), (3.16) and (3.27), we obtain

$$
\begin{aligned}
\left\|u\left(t, u_{0}\right)\right\|_{\mathrm{H}_{\alpha}} & =\left\|e^{t L_{1}} u_{0}+\int_{0}^{t} e^{(t-\tau) L_{1}} q_{1}(u, v, w, z) d \tau\right\|_{\mathrm{H}_{\alpha}} \\
& \leq\left\|e^{t L_{1}} u_{0}\right\|_{\mathrm{H}_{\alpha}}+\left\|\int_{0}^{t} e^{(t-\tau) L_{1}} q_{1}(u, v, w, z) d \tau\right\|_{\mathrm{H}_{\alpha}} \\
& \leq C\left\|u_{0}\right\|_{\mathrm{H}_{\alpha}}+\int_{0}^{t}\left\|\left(-L_{1}\right)^{\alpha-1} e^{(t-\tau) L_{1}}\right\| \cdot\left\|q_{1}(u, v, w, z)\right\|_{\mathrm{H}_{1}} d \tau \\
& \leq C\left\|u_{0}\right\|_{\mathrm{H}_{\alpha}}+C \int_{0}^{t} \tau^{1-\alpha} e^{-\delta \tau} d \tau \\
& \leq C, \forall t \geq 0, g_{0} \in B,
\end{aligned}
$$

where $1<\alpha<2$. By (3.2), (3.16) and (3.28), we obtain

$$
\begin{aligned}
\left\|v\left(t, v_{0}\right)\right\|_{\mathrm{H}_{\alpha}} & =\left\|e^{t L_{2}} v_{0}+\int_{0}^{t} e^{(t-\tau) L_{2}} q_{2}(u, v, w, z) d \tau\right\|_{\mathrm{H}_{\alpha}} \\
& \leq\left\|e^{t L_{2}} v_{0}\right\|_{\mathrm{H}_{\alpha}}+\left\|\int_{0}^{t} e^{(t-\tau) L_{2}} q_{2}(u, v, w, z) d \tau\right\|_{\mathrm{H}_{\alpha}} \\
& \leq C\left\|v_{0}\right\|_{\mathrm{H}_{\alpha}}+\int_{0}^{t}\left\|\left(-L_{2}\right)^{\alpha-1} e^{(t-\tau) L_{2}}\right\| \cdot\left\|q_{2}(u, v, w, z)\right\|_{\mathrm{H}_{1}} d \tau \\
& \leq C\left\|v_{0}\right\|_{\mathrm{H}_{\alpha}}+C \int_{0}^{t} \tau^{1-\alpha} e^{-\delta \tau} d \tau \\
& \leq C, \forall t \geq 0, g_{0} \in B,
\end{aligned}
$$

where $1<\alpha<2$. By (3.3), (3.4), (3.16), (3.29) and (3.30), simple calculations shows that

$$
\left\|w\left(t, w_{0}\right)\right\|_{\mathrm{H}_{\alpha}} \leq C, \forall t \geq 0, g_{0} \in B,
$$




$$
\left\|z\left(t, z_{0}\right)\right\|_{\mathrm{H}_{\alpha}} \leq C, \forall t \geq 0, g_{0} \in B
$$

where $1<\alpha<2$. By (3.31), (3.32), (3.33) and (3.34) together, we obtain (3.26) immediately.

In the same fashion as in the proof of (3.26), by iteration we can prove that for any bounded set $B \subset \mathcal{H}_{\alpha}$, there exists a positive constant $C$ such that

$\left\|u\left(t, u_{0}\right)\right\|_{\mathrm{H}_{\alpha}}^{2}+\left\|v\left(t, v_{0}\right)\right\|_{\mathrm{H}_{\alpha}}^{2}+\left\|w\left(t, w_{0}\right)\right\|_{\mathrm{H}_{\alpha}}^{2}+\left\|z\left(t, z_{0}\right)\right\|_{\mathrm{H}_{\alpha}}^{2} \leq C, \forall t \geq 0, \alpha \geq 0$.

It then follows from the above inequality that

$$
\left\|g\left(t, g_{0}\right)\right\|_{\mathcal{H}_{\alpha}} \leq C, \forall t \geq 0, \alpha \geq 0,
$$

that is, for all $\alpha \geq 0$, the solution $g=(u, v, w, z)$ of (1.1)-(1.6) is uniformly bounded in $\mathcal{H}_{\alpha}$.

Hence, Lemma 3.1 is proved.

Lemma 3.2. Suppose $d_{1}, d_{2}, F, k, D_{1}$ and $D_{2}$ are given positive parameters, $g=(u, v, w, z)$ is a solution to the problem (1.1)-(1.6), $g_{0}=\left(u_{0}, v_{0}, w_{0}, z_{0}\right) \in$ $\mathcal{H}_{\alpha}(\forall \alpha \geq 0)$, then, the problem (1.1)-(1.6) has a bounded absorbing set in $\mathcal{H}_{\alpha}$.

Proof. It suffices to prove that for any bounded set $B \subset \mathcal{H}_{\alpha}(\alpha \geq 0)$ with initial value $g_{0}=\left(u_{0}, v_{0}, w_{0}, z_{0}\right) \in B$, there exist $T>0$ and a constant $C>0$ independent of $\left(u_{0}, v_{0}, w_{0}, z_{0}\right)$, such that

$$
\left\|g\left(t, g_{0}\right)\right\|_{\mathcal{H}_{\alpha}} \leq C, \quad \forall t \geq T .
$$

Obviously, if we get

$$
\left\|u\left(t, u_{0}\right)\right\|_{\mathrm{H}_{\alpha}}^{2}+\left\|v\left(t, v_{0}\right)\right\|_{\mathrm{H}_{\alpha}}^{2}+\left\|w\left(t, w_{0}\right)\right\|_{\mathrm{H}_{\alpha}}^{2}+\left\|z\left(t, z_{0}\right)\right\|_{\mathrm{H}_{\alpha}}^{2} \leq C, \quad \forall t \geq T,
$$

then we obtain (3.35) immediately.

For $\alpha=\frac{1}{2}$, this follows from Propositions 2.5, 2.6 and 2.7. So we shall prove (3.35) for any $\alpha \geq \frac{1}{2}$. We prove the lemma in the following steps:

Step 1. we prove that for any $\frac{1}{2} \leq \alpha<1$, the problem (1.1)-(1.6) has a bounded absorbing set in $\mathcal{H}_{\alpha}$.

It then follows from (3.1)-(3.4) that

$$
\begin{gathered}
u\left(t, u_{0}\right)=e^{(t-T) L_{1}} u\left(T, u_{0}\right)+\int_{T}^{t} e^{(t-\tau) L_{1}} q_{1}(u, v, w, z) d \tau, \\
v\left(t, v_{0}\right)=e^{(t-T) L_{2}} v\left(T, v_{0}\right)+\int_{T}^{t} e^{(t-\tau) L_{2}} q_{2}(u, v, w, z) d \tau, \\
w\left(t, w_{0}\right)=e^{(t-T) L_{1}} w\left(T, w_{0}\right)+\int_{T}^{t} e^{(t-\tau) L_{1}} q_{3}(u, v, w, z) d \tau, \\
z\left(t, z_{0}\right)=e^{(t-T) L_{2}} z\left(T, z_{0}\right)+\int_{T}^{t} e^{(t-\tau) L_{2}} q_{4}(u, v, w, z) d \tau .
\end{gathered}
$$


Assume $\mathbb{B}$ is the bounded absorbing set of the problem (1.1)-(1.6) and satisfy $\mathbb{B} \subset \mathcal{H}$, we also assume $T_{0}>0$ the time such that $\forall t>T_{0},\left(u_{0}, v_{0}, w_{0}, z_{0}\right) \in$ $B \subset \mathcal{H}_{\alpha}$,

$$
\left(u\left(t, u_{0}\right), v\left(t, v_{0}\right), w\left(t, w_{0}\right), z\left(t, z_{0}\right)\right) \in \mathbb{B}, \quad \alpha \geq \frac{1}{2} .
$$

It is easy to check that

$$
\left\|e^{t L_{i}}\right\| \leq C e^{-d \lambda_{1} t},
$$

here, $i=1,2, \lambda_{1}>0$ is the first eigenvalue of the equation

$$
\left\{\begin{array}{l}
-\Delta \Sigma=\lambda \Sigma \\
\left.\frac{\partial \Sigma}{\partial \nu}\right|_{\partial \Omega}=0
\end{array}\right.
$$

where $\Sigma=u, v, w, z$.

Then, for any given $T>0$ and $\left(u_{0}, v_{0}, w_{0}, z_{0}\right) \in B \subset \mathcal{H}_{\alpha}\left(\alpha \geq \frac{1}{2}\right)$, we deduce that

$$
\begin{aligned}
& \lim _{t \rightarrow \infty}\left\|e^{(t-T) L_{1}} u\left(T, u_{0}\right)\right\|_{\mathrm{H}_{\alpha}}=0, \quad \lim _{t \rightarrow \infty}\left\|e^{(t-T) L_{2}} v\left(T, v_{0}\right)\right\|_{\mathrm{H}_{\alpha}}=0, \\
& \lim _{t \rightarrow \infty}\left\|e^{(t-T) L_{1}} w\left(T, w_{0}\right)\right\|_{\mathrm{H}_{\alpha}}=0, \quad \lim _{t \rightarrow \infty}\left\|e^{(t-T) L_{2}} z\left(T, z_{0}\right)\right\|_{\mathrm{H}_{\alpha}}=0 .
\end{aligned}
$$

Then, by (3.36) and (3.40), we obtain

$$
\begin{aligned}
& \left\|u\left(t, u_{0}\right)\right\|_{\mathrm{H}_{\alpha}} \\
\leq & \left\|e^{\left(t-T_{0}\right) L_{1}} u\left(T_{0}, u_{0}\right)\right\|_{\mathrm{H}_{\alpha}}+\int_{T_{0}}^{t}\left\|\left(-L_{1}\right)^{\alpha} e^{(t-\tau) L_{1}}\right\|\left\|q_{1}(u, v, w, z)\right\|_{\mathrm{H}} d \tau \\
\leq & \left\|e^{\left(t-T_{0}\right) L_{1}} u\left(T_{0}, u_{0}\right)\right\|_{\mathrm{H}_{\alpha}}+C \int_{T_{0}}^{t}\left\|\left(-L_{1}\right)^{\alpha} e^{(t-\tau) L_{1}}\right\| d \tau \\
\leq & \left\|e^{\left(t-T_{0}\right) L_{1}} u\left(T_{0}, u_{0}\right)\right\|_{\mathrm{H}_{\alpha}}+C \int_{0}^{T-T_{0}} \tau^{-\alpha} e^{-\delta \tau} d \tau \\
(3.43) \leq & \left\|e^{\left(t-T_{0}\right) L_{1}} u\left(T_{0}, u_{0}\right)\right\|_{\mathrm{H}_{\alpha}}+C_{1},
\end{aligned}
$$

where $C_{1}$ is a positive constant. By (3.37) and (3.40), we have

$$
\begin{aligned}
& \left\|v\left(t, v_{0}\right)\right\|_{\mathrm{H}_{\alpha}} \\
\leq & \left\|e^{\left(t-T_{0}\right) L_{2}} v\left(T_{0}, v_{0}\right)\right\|_{\mathrm{H}_{\alpha}}+\int_{T_{0}}^{t}\left\|\left(-L_{2}\right)^{\alpha} e^{(t-\tau) L_{2}}\right\|\left\|q_{2}(u, v, w, z)\right\|_{\mathrm{H}} d \tau \\
\leq & \left\|e^{\left(t-T_{0}\right) L_{2}} v\left(T_{0}, v_{0}\right)\right\|_{\mathrm{H}_{\alpha}}+C \int_{T_{0}}^{t}\left\|\left(-L_{2}\right)^{\alpha} e^{(t-\tau) L_{2}}\right\| d \tau \\
\leq & \left\|e^{\left(t-T_{0}\right) L_{2}} v\left(T_{0}, v_{0}\right)\right\|_{\mathrm{H}_{\alpha}}+C \int_{0}^{T-T_{0}} \tau^{-\alpha} e^{-\delta \tau} d \tau \\
(3.44) \leq & \left\|e^{\left(t-T_{0}\right) L_{2}} v\left(T_{0}, v_{0}\right)\right\|_{\mathrm{H}_{\alpha}}+C_{2},
\end{aligned}
$$

where $C_{2}$ is a positive constant. Using the same method, we can also obtain

$$
\left\|w\left(t, w_{0}\right)\right\|_{\mathrm{H}_{\alpha}} \leq\left\|e^{\left(t-T_{0}\right) L_{1}} w\left(T_{0}, w_{0}\right)\right\|_{\mathrm{H}_{\alpha}}+C_{3},
$$




$$
\left\|r\left(t, r_{0}\right)\right\|_{\mathrm{H}_{\alpha}} \leq\left\|e^{\left(t-T_{0}\right) L_{2}} r\left(T_{0}, r_{0}\right)\right\|_{\mathrm{H}_{\alpha}}+C_{4},
$$

where $C_{3}, C_{4}$ are positive constants.

Then, by (3.43)-(3.46), we obtain (3.35) holds for all $\frac{1}{2} \leq \alpha<1$.

Step 2. We prove that for any $1 \leq \alpha<\frac{3}{2}$, the problem (1.1)-(1.6) has a bounded absorbing set in $\mathcal{H}_{\alpha}$.

By (3.36) and (3.18), we obtain

$$
\begin{aligned}
& \left\|u\left(t, u_{0}\right)\right\|_{\mathrm{H}_{\alpha}} \\
\leq & \left\|e^{\left(t-T_{0}\right) L_{1}} u\left(T_{0}, u_{0}\right)\right\|_{\mathrm{H}_{\alpha}}+\int_{T_{0}}^{t}\left\|\left(-L_{1}\right)^{\alpha-\frac{1}{2}} e^{(t-\tau) L_{1}}\right\|\left\|q_{1}(u, v, w, z)\right\|_{\mathrm{H}_{\frac{1}{2}}} d \tau \\
\leq & \left\|e^{\left(t-T_{0}\right) L_{1}} u\left(t, u_{0}\right)\right\|_{\mathrm{H}_{\alpha}}+C \int_{T_{0}}^{t}\left\|\left(-L_{1}\right)^{\alpha-\frac{1}{2}} e^{(t-\tau) L_{1}}\right\| d \tau \\
\leq & \left\|e^{\left(t-T_{0}\right) L_{1}} u\left(T_{0}, u_{0}\right)\right\|_{\mathrm{H}_{\alpha}}+C \int_{T_{0}}^{t} \tau^{-\left(\alpha-\frac{1}{2}\right)} e^{-\delta \tau} d \tau \\
\leq & \left\|e^{\left(t-T_{0}\right) L_{1}} u\left(T_{0}, u_{0}\right)\right\|_{\mathrm{H}_{\alpha}}+C_{5},
\end{aligned}
$$

where $C_{5}$ is a positive constant. By (3.37) and (3.19), we have

(3.48) $\left\|v\left(t, v_{0}\right)\right\|_{\mathrm{H}_{\alpha}}$

$$
\begin{aligned}
& \leq\left\|e^{\left(t-T_{0}\right) L_{2}} v\left(T_{0}, v_{0}\right)\right\|_{\mathrm{H}_{\alpha}}+\int_{T_{0}}^{t}\left\|\left(-L_{2}\right)^{\alpha-\frac{1}{2}} e^{(t-\tau) L_{2}}\right\|\left\|q_{2}(u, v, w, z)\right\|_{\mathrm{H}_{\frac{1}{2}}} d \tau \\
& \leq\left\|e^{\left(t-T_{0}\right) L_{2}} v\left(t, v_{0}\right)\right\|_{\mathrm{H}_{\alpha}}+C \int_{T_{0}}^{t}\left\|\left(-L_{2}\right)^{\alpha-\frac{1}{2}} e^{(t-\tau) L_{2}}\right\| d \tau \\
& \leq\left\|e^{\left(t-T_{0}\right) L_{2}} v\left(T_{0}, v_{0}\right)\right\|_{\mathrm{H}_{\alpha}}+C \int_{T_{0}}^{t} \tau^{-\left(\alpha-\frac{1}{2}\right)} e^{-\delta \tau} d \tau \\
& \leq\left\|e^{\left(t-T_{0}\right) L_{2}} v\left(T_{0}, v_{0}\right)\right\|_{\mathrm{H}_{\alpha}}+C_{6}
\end{aligned}
$$

where $C_{6}$ is a positive constant. Using the same method, we can also obtain

$$
\begin{aligned}
\left\|w\left(t, w_{0}\right)\right\|_{\mathrm{H}_{\alpha}} & \leq\left\|e^{\left(t-T_{0}\right) L_{1}} w\left(T_{0}, w_{0}\right)\right\|_{\mathrm{H}_{\alpha}}+C_{7}, \\
\left\|z\left(t, z_{0}\right)\right\|_{\mathrm{H}_{\alpha}} & \leq\left\|e^{\left(t-T_{0}\right) L_{2}} z\left(T_{0}, z_{0}\right)\right\|_{\mathrm{H}_{\alpha}}+C_{8}
\end{aligned}
$$

where $C_{7}, C_{8}$ are positive constants.

Then, by (3.47)-(3.50), we obtain (3.35) holds for all $1 \leq \alpha<\frac{3}{2}$.

By iteration, we can prove that for any $\alpha \geq \frac{3}{2}$, (3.35) holds. Therefore, the problem (1.1) has a bounded absorbing set in $\mathcal{H}_{\alpha}$.

Then, Lemma 3.2 is proved.

Now, we give the proof the main result.

Proof of Theorem 2.10. By Lemma 2.1, Lemma 3.1, Lemma 3.2, we immediately conclude that the proof of Theorem 2.10 is completed. 
Acknowledgements. The authors would like to thank the referees for the valuable comments and suggestions about this paper.

\section{References}

[1] M. Ashkenazi and H. G. Othmer, Spatial patterns in coupled biochemical oscillators, J. Math. Biol. 5 (1978), no. 4, 305-350.

[2] J. F. G. Auchmuty and G. Nicolis, Bifurcation analysis of nonlinear reaction-diffusion equations I: evolution equations and the steady state solutions, Bull. Math. Biol. 37 (1975), no. 4, 323-365.

[3] T. Dlotko, Global attractor for the Cahn-Hilliard equation in $H^{2}$ and $H^{3}$, J. Differential Equations 113 (1994), no. 2, 381-393.

[4] P. Gormley, K. Li, and G. W. Irwin, Modeling molecular interaction pathways using a two-stage identification algorithm, Syst. Synth. Biol. 1 (2007), no. 3, 145-160.

[5] P. Gray and S. K. Scott, Autocatalytic reactions in the isothermal continuous stirred tank reactor: isolas and other forms of multistability, Chem. Eng. Sci. 38 (1983), 29-43.

[6] - Autocatalytic reactions in the isothermal, continuous stirred tank reactor: oscillations and instabilities in the system $A+2 B \rightarrow 3 B, B \rightarrow C$, Chem. Eng. Sci. 39 (1984), 1087-1097.

[7] _ Chemical Waves and Instabilities, Clarendon, Oxford, 1990.

[8] J. K. Hale, L. A. Peletier, and W. C. Troy, Exact homoclinic and heteroclinic solutions of the Gray-Scott model for autocatalysis, SIAM J. Appl. Math. 61 (2000), no. 1, 102-130.

[9] M. Kawato and R. Suzuki, Two coupled neural oscillators as a model of the circadian pacemaker, J. Theoret. Biol. 86 (1980), no. 3, 547-575.

[10] K. J. Lee, W. D. McCormick, J. E. Pearson, and H. L. Swinney, Experimental observations of self-replicating spots in a reaction-diffusion system, Nature 369 (1994), $215-218$.

[11] D. S. Li and C. K. Zhong, Global attractor for the Cahn-Hilliard system with fast growing nonlinearity, J. Differential Equations 149 (1998), no. 2, 191-210.

[12] T. Ma and S. H. Wang, Stability and Bifurcation of Nonlinear Evolution Equations, Science Press, Beijing, 2006, (in Chinese).

[13] J. S. McGough and K. Riley, Pattern formation in the Gray-Scott model, Nonlinear Anal. Real World Appl. 5 (2004), no. 1, 105-121.

[14] Y. Nishiura and D. Ueyama, Spatio-temporal chaos for the Gray-Scott model, Phys. D 150 (2001), 137-162.

[15] I. Schreiber and M. Marek, Strange attractors in coupled reaction-diffusion cells, Phys. D 5 (1982), no. 2-3, 258-272.

[16] G. R. Sell and Y. You, Dynamics of Evolutionary Equations, Springer, New York, 2002.

[17] L. Song, Y. Zhang, and T. Ma, Global attractor of the Cahn-Hilliard euqation in $H^{k}$ spaces, J. Math. Anal. Appl. 355 (2009), no. 1, 53-62.

[18] Global attractor of a modified Swift-Hohenberg equation in $H^{k}$ space, Nonlinear Anal. 72 (2010), no. 1, 183-191.

19] R. Temam, Infinite-Dimensional Dynamical Systems in Mechanics and Physics, Springer-Verlag, New York, 1988.

[20] Y. You, Dynamics of two-compartment Gray-Scott equations, Nonlinear Anal. 74 (2011), no. 5, 1969-1986.

[21] _ Global dynamics of a reaction-diffusion system, Electron. J. Differential Equations 2011 (2011), no. 25, 1-28.

[22] X. Zhao and B. Liu, The existence of global attractor for convective Cahn-Hilliard equation, J. Korean Math. Soc. 49 (2012), no. 2, 357-378.

[23] X. Zhao and C. Liu, The existence of global attractor for a fourth-order parabolic equation, Appl. Anal. 92 (2013), no. 1, 44-59. 
[24] S. Zheng and A. Milani, Global attractors for singular perturbations of the Cahn-Hilliard equations, J. Differential Equations 209 (2005), no. 1, 101-139.

XiaOpeng ZhaO

College of Mathematics

JiLIN UNIVERSITY

Changchun, 130012, P. R. China

E-mail address: zxp032@gmail.com

Bo LiU

College of Mathematics

JILIN UNIVERSITY

Changchun, 130012, P. R. China

E-mail address: liubom@jlu.edu.cn 